\title{
A Novel DGS to Bandpass Filter Design
}

\author{
Hongqing $\mathrm{He}^{\mathrm{a}}$, Minquan $\mathrm{Li}^{\mathrm{b}}$, Yu Zhang ${ }^{\mathrm{c}}$ \\ Key Laboratory of Intelligent Computing \& Signal Processing, Ministry of Education, Anhui University, \\ Hefei 230039, China. \\ ahqhemeet@163.com, ${ }^{\text {b }}$ limq@ahu.edu.cn, c soaptesla@gmail.com
}

Keywords: bandpass filter, defected ground structure, miniaturization, split-ring resonator.

\begin{abstract}
In this paper, a novel symmetrical split-ring resonators defected ground structure (DGS) wideband bandpass filter is proposed. This filter exhibits advantages such as miniaturization, large range of bandwidth and easy fabrication. This paper analyzes the symmetrical split-ring resonators DGS unit and the bandpass filter with three symmetrical split-ring resonators DGS is designed. The simulation and testing show that the bandpass filter with the novel DGS provides insertion loss of $0.9 \mathrm{~dB}$, return loss of $-12.5 \mathrm{~dB}$, and the relative bandwidth achieves $7 \%$.
\end{abstract}

\section{Introduction}

Defected Ground Structure (DGS) is first proposed on the basis of discussing Photonic Band Gap(PBG) Structure by south Korean scholars J. I. Park and C. S. Kim et.al in 1999 [1,2]. It can be modeled with a parallel L-C resonant circuit and the parameter extraction is simple. The resonance frequency can be directly derived from the physical structure of the DGS [3]. The current of ground can be changed by etching different patterns on the ground. Therefore the distributed capacitance and inductance of the microstrip line is adjustable. Generally, DGS can make the band rejection and slow-wave characteristics [4]. Moreover, the DGS has the advantages such as simple structure, low power consumption, etc[5].The DGS is advantageous in the design of microwave and millimeter-wave circuits.

In this paper, we propose a novel DGS which has three symmetrical split-ring resonators on the ground plane. Compared with the conventional DGS [6-8], the novel DGS we proposed can provide two transmission zeros and a wide stopband. Based on the property of the novel DGS, the bandpass filter utilizing the novel symmetrical split-ring resonators DGS can be designed. Analysis and adjusting the geometric properties of the DGS, the transmission poles of the bandpass filter are changed. Meanwhile, the relative bandwidth and insertion loss get much improvement. Finally, wideband bandpass filter is optimally designed and fabricated.

\section{The symmetrical split-ring resonators DGS design}

In Fig. 1(a), the geometry of symmetrical split-ring resonators DGS on the ground plane of the microstrip line is shown, where the dimensions of a symmetrical split-ring resonators defects on the right and left hand sides are identical. The equivalent circuit model of the DGS is shown in Fig. 1(b). The S-parameters (S21) in Fig.2 shows that the symmetrical split-ring resonators DGS can provide two reflection zeros and a wide stopband.

In order to discuss the transfer characteristics of the DGS, the symmetrical split-ring resonators DGS on the ground plane of the microstrip line is researched by changing the length of split-ring (l) and opening width(m1) respectively.

The different resonance frequencies (fs) of the DGS with various length of split-ring (l) are shown in Fig.3 (a). It's obviously that as 1 increases, the resonance frequency (fs) decreases. As l values increases, the equivalent parallel inductance (L1) increases, the equivalent parallel capacitor (C1) decreases, and the resonance frequency (fs) moves to a lower band. It is noted that as l values increases, the equivalent inductance (L2) of transmission line and equivalent capacitor (C2) increases. 
The resonance frequency (fs) of the DGS with different opening width(m1) are illustrated in Fig. 3 (b).As $\mathrm{m} 1$ values increases, the resonance frequency (fs) increases, obviously. As $\mathrm{m} 1$ values increases, the equivalent parallel inductance (L1) decreases, the equivalent parallel capacitor (C1) increases, so that the resonance frequency (fs) moves to a higher band. However, as $\mathrm{m} 1$ values increases, the equivalent inductance (L2) of transmission line and equivalent capacitor (C2) remain the same.

Through analyzing the symmetrical split-ring resonators DGS, the resonance frequency (fs) of the split-ring resonators is decided by the equivalent parallel inductance (L1), the equivalent parallel capacitor (C1) and the equivalent capacitor (C2) of transmission line. In addition, the resonance frequency $\left(f_{s}\right)$ determines the position of transmission zeros, which is provided by DGS. The resonance frequency $\left(f_{s}\right)$ is given by:

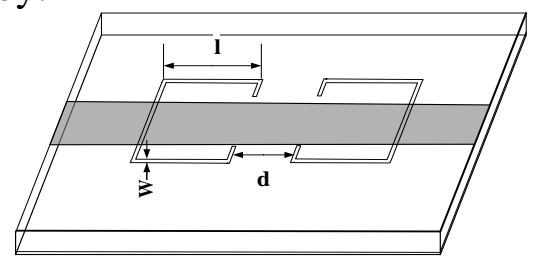

(a)

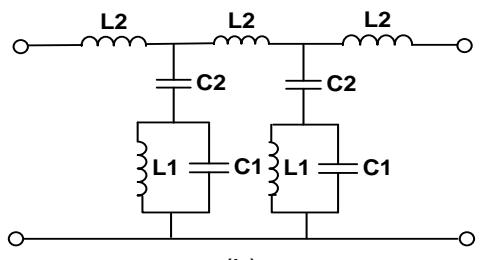

(b)

Fig.1 (a) Microstrip line with symmetrical split-ring resonators DGS on the ground and (b) equivalent circuit of the DGS.

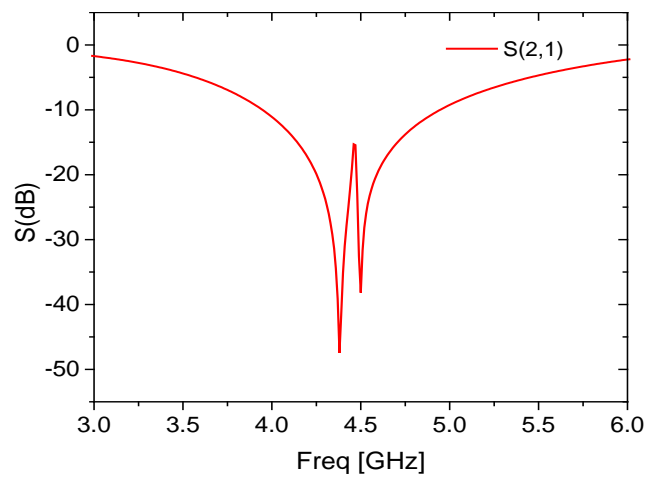

Fig.2 S parameters of the DGS
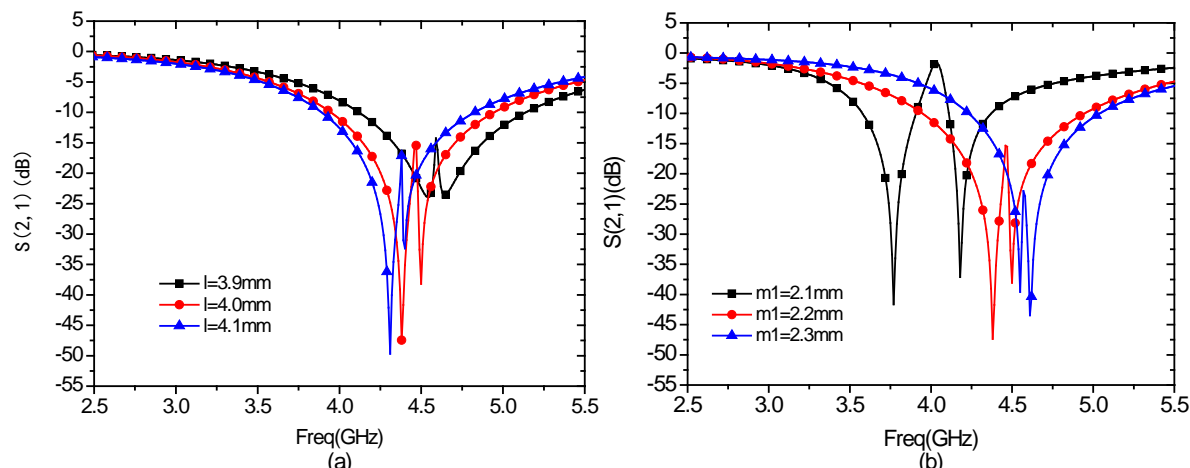

Fig.3 The characteristic curve of DGS changed with (a) the length of split-ring (l) and (b) opening width (m1)

$$
f_{s}=\frac{1}{2 \pi \sqrt{L_{1}\left(C_{1}+C_{2}\right)}}
$$


The impedance of L-C equivalent circuit equals to the impedance of Butterworth low-pass filter, so equivalent capacitance and equivalent inductance can be obtained. The circuit parameters of the equivalent circuit are extracted from the simulated scattering parameters [9]:

$$
\begin{aligned}
& C 1=\frac{\omega_{0}}{Z_{0} g_{1}} \cdot \frac{1}{\omega_{0}^{2}-\omega_{c}^{2}} \\
& L 1=\frac{1}{4 \pi^{2} f_{0}^{2} C}
\end{aligned}
$$

Here, $f_{0}$ is the resonance frequency of Butterworth low-pass filter, $\omega_{0}$ is the 3-dB cutoff frequency, and $\omega_{0}$ is the impedance, and $\mathrm{Z} 0$ is the characteristic impedance of the microstrip line.

\section{The bandpass filter design}

Fig. 4(a) illustrates the geometry of the bandpass filter. The optimum dimensions of the filter are listed as follows: $\mathrm{t}=6.1 \mathrm{~mm}, \mathrm{f}=0.1 \mathrm{~mm}, \mathrm{p}=1.25 \mathrm{~mm}, \mathrm{e}=1.25 \mathrm{~mm}, \mathrm{~h}=1.5 \mathrm{~mm}$. This structure is etched on the substrate material (Rogers RO3210) with permittivity of 10.2 and thickness of $0.8 \mathrm{~mm}$. This filter is simulated by the high frequency simulation software HFSS 13.0, the S-parameters are shown in Fig. 4(b), the center frequency and insertion loss is $5.7 \mathrm{GHz}$ and $1.0 \mathrm{~dB}$ respectively, the return loss is $-23 \mathrm{~dB}$.

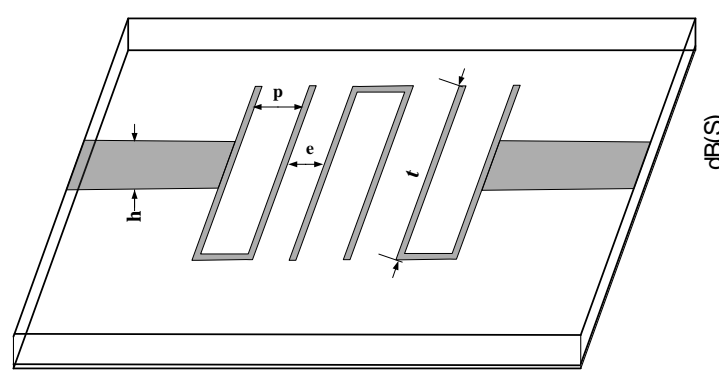

(a)

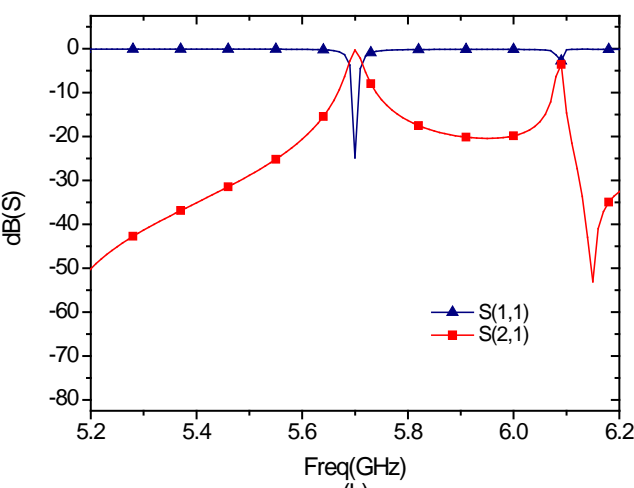

(b)

Fig.4 (a) the bandpass filter. (b) S parameters of the bandpass filter

Since the symmetrical split-ring resonators DGS has band rejection characteristic, it can be utilized in bandpass filter design. The bandpass filter with symmetrical split-ring resonators DGS is shown in Fig.5 (a). The concrete sizes are listed as follows: $w=0.17 \mathrm{~mm}, \mathrm{l}=4 \mathrm{~mm}, \mathrm{~d}=2.2 \mathrm{~mm}$, $\mathrm{m} 1=2.2 \mathrm{~mm}$. The S-parameters are illustrated in Fig.5 (b), the center frequency of the filter is $4.53 \mathrm{GHz}$, the insertion loss is $3.4 \mathrm{~dB}$, return loss is $-13.8 \mathrm{~dB}$, the relative bandwidth achieve $2 \%$. Since etching the DGS, the band rejection and slow-wave characteristics are introduced. It makes the transmission poles of the bandpass filter change. However, the bandpass filter with single DGS has a narrow pass band and the insertion loss is bad.

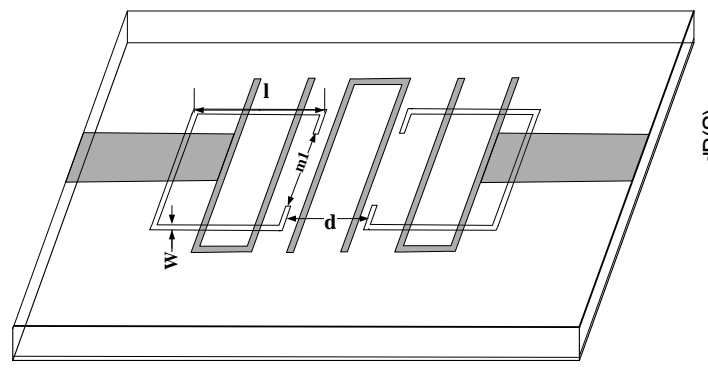

(a)

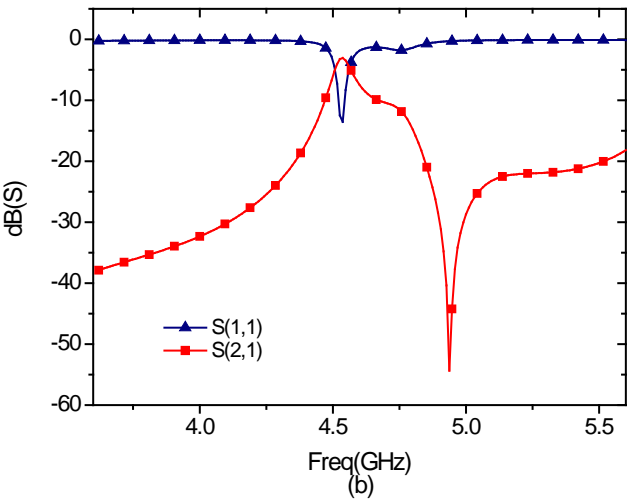

Fig.5 (a) the bandpass filter with the DGS. (b) S parameters of the bandpass filter with the DGS 
In order to overcome the limitation the filter with single DGS, the three symmetrical split-ring resonators DGS is proposed, the geometry shape is illustrated in Fig.6 (a). The equivalent circuit model of the filter is shown in Fig.6 (b). The concrete sizes are listed as follows: $\mathrm{m} 2=0.2 \mathrm{~mm}$, $\mathrm{m} 3=0.45 \mathrm{~mm}$.

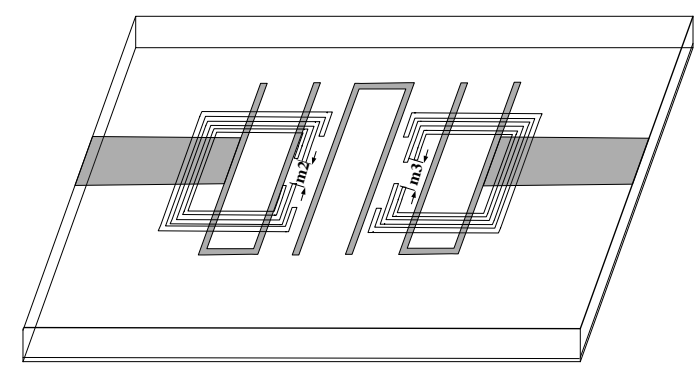

(a)

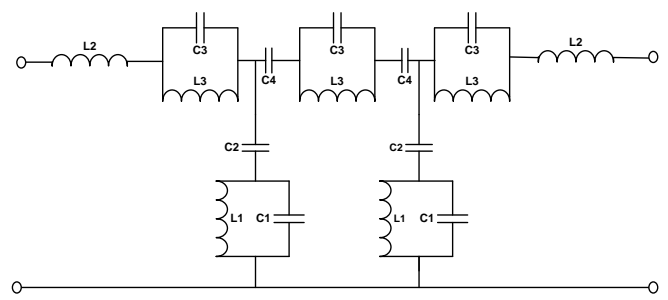

(b)

Fig.6 (a) the bandpass filter with three DGS. (b)Equivalent circuit of the bandpass filter with three DGS

The bandpass filter with the three symmetrical split-ring resonators DGS is simulated comprehensively, and the photograph of the fabricated bandpass filter is shown in Fig.7. The measurement is carried out by using E8363C PNA vector network analyzer. Measured and simulated results are shown in Fig.8, clearly one basic match. As can be seen from Fig.8, two reflection poles have increased and relative bandwidth increased to 7\%. In addition, compared with the filter with single DGS, the insertion loss have been greatly improved. The insertion loss of this bandpass filter is reduced to $0.9 \mathrm{~dB}$ and return loss is $-12.5 \mathrm{~dB}$.
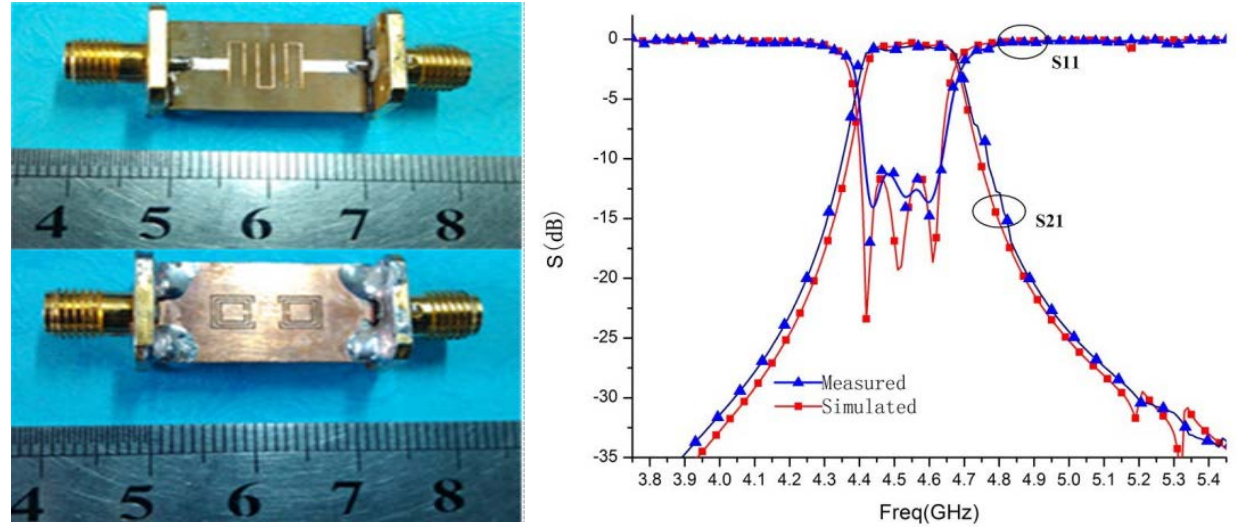

Fig.7 Photograph of the fabricated filter Fig.8 Comparison of the simulated and measured results

\section{Conclusions}

In this paper, a novel symmetrical split-ring resonator DGS is proposed. The transfer characteristics of the DGS have been discussed with the change in the parameters of DGS. The bandpass filter with three symmetrical split-ring resonators DGS have been designed and fabricated. The research shows that the DGS can improve the relative bandwidth, insertion loss and reduce geometry sizes effectively. The filter based on the DGS provide insertion loss of $0.9 \mathrm{~dB}$, return loss of $-12.5 \mathrm{~dB}$ and the relative bandwidth achieve $7 \%$. The proposed structures may have wide applications in the microwave and millimeter wave circuits. 


\section{References}

[1] Ahn D, Park J S, Kim J, et al. A design of the low-pass filter using the novel microstrip defected ground structure. IEEE Trans Microwave Theory Tech.Vol.49 (2001) No. 9, p. 83-93.

[2] Park J I, Kim C S, Kim J, et al. Modeling of a photonic bandgap and its application for the low-pass filter design. Asia Pacific Microwave Conference. Singapore, 1999, p.331 - 334.

[3] Anak Agung Alit Apriyana, Yue Ping Zhang,Joseph S. Chang, et al .Single-pole multiple-throw switches with defected ground structure low-pass filter. IET Microwaves, Antennas \& Propagation.Vol.8 (2014) No. 14, p.1241-1249.

[4] Yu-nan Han, Biao Yang, Qing Guo. Design of Low-Pass Filters with Ultra-wide Stopband Using Asymmetric DGS.IEEE International Symposium Electromagnetic Compatibility (EMC).2013, p.636 - 639.

[5] Lim J S,Kim H S,Park J S,et al. Vertically periodic defected ground structure for planar transmission lines. ElectronicsLett. Vol.38 (2002) No.15, p.803-804.

[6] J.-C. Liu, D.-S. Shu, B.-H. Zeng, et al. Improved equivalent circuits for complementary split-ring resonator-based high-pass filter with C-shaped couplings. Microwaves, Antennas \& Propagation, IET. Vol.2 (2008) No.6, p.622-626.

[7] Riana H. Geschke, Branka Jokanovic, Petrie Meyer. Filter Parameter Extraction for Triple-Band Composite Split-Ring Resonators and Filters. Microwave Theory and Techniques. Vol. 59 (2011) No.6, p.1500-1508.

[8] Yang Cai, Zuping Qian, Wenquan Cao, et al. HMSIW bandstop filter loaded with half complementary split-ring resonator [J]. Electronics Letters, 2015, 51(8):632-633.

[9] Ahn D, Park J I, Kim C S, Kim J, et al. A Design of the Low-pass Filter Using the Novel Microstrip Defected Ground Structure. IEEE Trans Microwave Theory and Techniques. Vol.49 (2001) No.1, p.86-93. 